\title{
ACOLHIMENTO DA EQUIPE DE ENFERMAGEM FRENTE AOS ADOLESCENTES COM IDEAÇÕES SUICIDAS: UMA REVISÃO INTEGRATIVA DE LITERATURA
}

\section{REVISÃO INTEGRATIVA}

NUNES, Nandara Rocha ${ }^{1}$

FREIRE, William Fernandes ${ }^{2}$

PORFIRIO, Regiane Baptista Martins ${ }^{3}$

NUNES, Nandara Rocha. FREIRE, William Fernandes. PORFIRIO, Regiane Baptista Martins. Acolhimento da equipe de enfermagem frente aos adolescentes com ideações suicidas: uma revisão integrativa de literatura. Revista Científica Multidisciplinar Núcleo do Conhecimento. Ano 04, Ed. 11, Vol. 03, pp. 49-66. Novembro de 2019. ISSN: 2448-0959, Link de acesso: https://www.nucleodoconhecimento.com.br/saude/ideacoes-suicidas

\section{RESUMO}

Introdução: No Brasil a incidência de suicídios têm aumentado gradativamente nos últimos anos, sendo a segunda maior causa de morte entre os adolescentes. Os dados levantados permitiram destacar os fatores que estão associados à ideação suicida $e$ às tentativas de suicídios. Esse estudo visou identificar o tipo de abordagem que deve ser direcionada aos pacientes suicidas, e como isso pode influenciar na recidiva do

\footnotetext{
${ }^{1}$ Graduanda em Enfermagem na Universidade Anhembi Morumbi.

2 Socorrista e Graduando Em Enfermagem na Universidade Anhembi Morumbi.

${ }^{3}$ Doutorado em Enfermagem. Mestrado em Pós Graduação Stricto Sensu na Área da Saúde do Ad. Especialização em Gerenciamento em Serviços de Enfermagem. Especialização em Pós Graduação Lato Sensu Em Saúde Pública. Graduação em Enfermagem Bacharelado.
} 
ato. Objetivo: Constatar na literatura a existência do acolhimento efetivo, humanizado e qualificado da equipe de enfermagem para com os adolescentes que possuem ideações suicidas ou que já tentaram o suicídio. Método: Revisão integrativa de literatura com caráter descritivo, foram selecionados os artigos publicados entre os anos 2012 a 2019, sendo eles em língua portuguesa, inglesa ou espanhola. Inicialmente foram obtidos 22 artigos, após análises criteriosas selecionamos 14 deles por possuírem mais correlação com a problemática discutida. Resultados: No Brasil ocorreram 11.947 mortes por lesões autoprovocadas intencionalmente em indivíduos de 10 a 19 anos no período de 2000 a 2015 . A maior parte (85,32\%) dos suicídios na faixa etária estudada aconteceu com adolescentes de 15 a 19 anos. Os fatores associados à ideação suicida na adolescência são multifacetados e aqueles que mais sobressaem são: Depressão, desesperança, solidão, tristeza, preocupação, ansiedade, baixa autoestima, uso de substâncias, agressões, entre outros. Conclusão: O número de adolescentes com inclinação para a ideação suicida está em constante crescimento nos últimos anos. Devido a isso, os profissionais de enfermagem devem estar prontos para identificar e prestar assistência integral para esses adolescentes, garantindo um atendimento humanizado, por meio da escuta ativa, autorreflexão e comunicação terapêutica.

Palavras-chave: Ideação suicida, adolescente, acolhimento, enfermagem.

\section{INTRODUÇÃO}

O suicídio, na atualidade, é considerado um problema significativo de saúde pública. No Brasil, a taxa de suicídio aumentou cerca de $27 \%$ nos últimos anos (BARROS, 2018), e recentemente tem sido notada maior prevalência de atos suicidas entre jovens (FILHO e ZERBINI, 2016).

A morte é temida pela maioria das pessoas, independente da faixa etária; contudo, pode ser considerada como a única alternativa para aqueles que não encontram soluções para seus problemas, o que leva a pessoa por fim na sua vida (MOREIRA e BASTOS, 2015). 
A cada ano, cerca de 800 mil pessoas tiram a própria vida e um número ainda maior de indivíduos tentam suicídio. Cada suicídio é uma tragédia que afeta famílias, comunidades e países inteiros e tem efeitos duradouros sobre as pessoas deixadas para trás. Segundo a Organização Mundial de Saúde (OMS), o suicídio ocorre durante todo o curso de vida do ser humano e foi a segunda principal causa de morte entre jovens de 15 a 29 anos em todo o mundo no ano de 2016. Índices maiores são encontrados entre os adolescentes em comparação com qualquer outra idade (OMS, 2018).

Além disso, para cada suicídio em adolescentes ocorridos no mundo, existem, pelo menos 40 tentativas não fatais, segundo a OMS (2018). Tal fato justifica-se pelo confronto de adolescentes, na sociedade atual, com inúmeros componentes estressores da vida diária, características do seu estágio de desenvolvimento (BLUTH e BLANTON, 2013).

Destaca-se que a adolescência, fase compreendida entre 12 e 18 anos de idade, é caracterizada por um período de desenvolvimento marcado por diversas modificações biológicas, psicológicas e sociais que, geralmente, são acompanhadas de conflitos e angústias, tem-se observado, nas últimas décadas, um crescimento no comportamento suicida entre jovens (BREDA e GUERRA, 2019).

Para muitos adolescentes, as necessidades de ajustes e reajustes internos e externos, a instabilidade e o desequilíbrio, geram dificuldades no desenvolvimento saudável nos campos afetivo, pessoal, familiar, escolar e de socialização, tornandoos desta forma vulneráveis a comportamentos autolesivos (MOREIRA e BASTOS, 2015).

O comportamento suicida pode ser classificado em três momentos: a ideação suicida (que pode ir de pensamentos de morte à intenção suicida estruturada com ou sem planejamento suicida), o suicídio consumado e a tentativa de suicídio que acontece entre a ideação e o suicídio consumado (NAVA et al., 2019). 
Para aqueles adolescentes que possuem pensamento suicida ou tentam suicídio sem sucesso, há a necessidade de serem acolhidos por familiares e profissionais da saúde.

De acordo com o dicionário Aurélio de Língua Portuguesa, o termo acolhimento está relacionado ao "ato ou efeito de acolher; recepção, atenção, consideração, refúgio, abrigo, agasalho". Na enfermagem, o acolhimento engloba a humanidade dentro do atendimento realizado com dignidade a quem está precisando de ajuda, ao mesmo tempo que os profissionais asseguram a esses pacientes da efetividade positiva do vínculo que está sendo construído, oferecendo compreensão e possibilidade de encontrarem soluções para os problemas levados (COSTA, GARCIA, TOLEDO, 2016).

\section{OBJETIVO}

Identificar, na literatura nacional e internacional, experiências relatadas por enfermeiros relacionadas com o acolhimento humanizado, qualificado e efetivo, direcionado aos adolescentes com ideações suicidas ou que tentaram suicídio.

\section{METODOLOGIA}

\subsection{TIPO DE ESTUDO}

Trata-se de uma revisão integrativa de literatura, com caráter descritivo, que é um método de revisão específico que reúne achados de estudos desenvolvidos mediante diferentes metodologias e requer que os revisores procedam à análise e à síntese dos dados primários de forma sistemática e rigorosa (SOARES et al., 2014).

\subsection{OPERACIONALIZAÇÃO DA COLETA DE DADOS}

A pesquisa foi realizada no período de janeiro a setembro de 2019. Os estudos incluídos na presente revisão integrativa obedeceram aos seguintes critérios de inclusão: resumo disponível nas bases de dados; publicados entre os anos de 2012 a 2019; língua portuguesa, inglesa e espanhola; temática pertinente ao acolhimento da 
equipe de enfermagem junto ao adolescente com ideação suicida ou que tentou suicídio. Foram encontrados 22 artigos cujos resumos foram lidos e selecionados 14 que tinham correlação com a temática desta pesquisa.

A coleta de dados ocorreu no período de junho a outubro de 2019, baseada nas palavras chave indexadas nos Descritores em Ciências da Saúde (DeCS): Ideação Suicida; Adolescente; Acolhimento; Enfermagem. As bases de dados utilizadas foram: Literatura Latino-Americana em Ciências de Saúde (LILACS), Scientific Electronic Library Online (SCIELO), Medical Literature Analysis and Retrieval System Online (MEDLINE), Portal Regional da Biblioteca Virtual em Saúde (BVS), Centro LatinoAmericano e do Caribe de Informação em Ciências da Saúde (BIREME), Fundación Dialnet - Universidad de La Rioja (DIALNET) e Red de Revistas Científicas de América Latina y el Caribe, España y Portugal (REDALYC) (Tabela 1).

A revisão foi ampliada por meio de outras fontes (Tabela 1) como:

- www.revistas.usp.br/sej/;

- www.arca.fiocruz.br;

- www.revista.ufpi.br/;

- www.revista.unilus.edu.br;

- www.editorarealize.com.br/revistas/conbracis/.

Os autores desse estudo partiram da seguinte pergunta norteadora: $O$ atendimento da equipe de enfermagem é acolhedor junto aos adolescentes com ideações suicidas ou que tentaram suicídio?

Tabela 1 - Distribuição das bases de dados utilizadas para levantamento dos artigos científicos, 2019.

\begin{tabular}{|l|l|l|}
\hline FONTES & $\mathrm{N}$ & $\%$ \\
\hline LILACS & 1 & 7,14 \\
\hline SCIELO & 3 & 21,43 \\
\hline MEDLINE & 2 & 14,29 \\
\hline
\end{tabular}




\begin{tabular}{|l|l|l|}
\hline Portal Regional da BVS & 1 & 7,14 \\
\hline BIREME & 1 & 7,14 \\
\hline DIALNET & 1 & 7,14 \\
\hline PORTAL DE REVISTAS DA USP & 1 & 7,14 \\
\hline $\begin{array}{l}\text { ARCA - REPOSITÓRIO INSTITUCIONAL DA FIOCRUZ } \\
\text { REVISTA DE ENFERMAGEM DA UFPI }\end{array}$ & 1 & 7,14 \\
\hline $\begin{array}{l}\text { REVISTA UNILUS ENSINO \& PESQUISA } \\
\text { CONBRACIS - ANAIS DO III CONGRESSO BRASILEIRO DE } \\
\text { CIÊNCIAS DA SAÚDE }\end{array}$ & 1 & $\mathbf{1}$ \\
\hline TOTAL & $\mathbf{1 4}$ & $\mathbf{1 0 0}$ \\
\hline
\end{tabular}

Fonte: Elaborada pelos próprios autores desse estudo.

Não houve nenhum tipo de financiamento ou benefícios recebidos de fontes comerciais, ou quaisquer conflitos de interesse que comprometa o trabalho apresentado.

\section{RESULTADOS}

A partir da busca digital, foram localizados 22 artigos dos quais 14 atenderam a todos os critérios de inclusão. Os resultados mais relevantes como título do artigo, autores, ano de publicação e síntese do estudo relacionados com os 14 artigos estão organizados em formato de quadro para melhor visualização (Quadro 1).

Quadro 1 - Resultados da síntese dos artigos selecionados na revisão de literatura.

\begin{tabular}{|c|c|c|}
\hline TÍTULO DO ARTIGO & SÍNTESE DO ESTUDO & AUTORES/ANO \\
\hline $\begin{array}{l}\text { Comportamento } \\
\text { Suicida: Um Olhar para }\end{array}$ & $\begin{array}{l}\text { O enfermeiro tem a } \\
\text { oportunidade de manter }\end{array}$ & $\begin{array}{lll}\text { VABO, A. } & \text { S. } & \text { R.; } \\
\text { CONRAD, } & & \text { D.; }\end{array}$ \\
\hline $\begin{array}{l}\text { Além do Modelo } \\
\text { Biomédico. }\end{array}$ & $\begin{array}{l}\text { proximidade ao cuidado } \\
\text { prestado, possibilitando a } \\
\text { utilização de ferramentas que } \\
\text { favoreçam abordagens }\end{array}$ & $\begin{array}{ll}\text { BAPTISTA, } & \text { C.; } \\
\text { AGUIAR, B. G. } & \text { C.; }\end{array}$ \\
\hline
\end{tabular}




\begin{tabular}{|c|c|c|}
\hline & $\begin{array}{l}\text { assertivas na prevenção ao } \\
\text { comportamento suicida. Por } \\
\text { meio da identificação e } \\
\text { entendimento das ações de } \\
\text { saúde que permitam a } \\
\text { valorização da vida e a } \\
\text { compreensão do sofrimento } \\
\text { experimentado pelo outro. }\end{array}$ & $\begin{array}{l}\text { FREITAS, V. } \\
\text { PEREIRA, G. L. } \\
(2016)\end{array}$ \\
\hline $\begin{array}{l}\text { A Atuação do } \\
\text { Enfermeiro com a } \\
\text { Pessoa em Situação } \\
\text { de Suicídio: Análise } \\
\text { Reflexiva. }\end{array}$ & $\begin{array}{l}\text { O enfermeiro e equipe de } \\
\text { saúde devem ser qualificados e } \\
\text { trabalharem em rede com as } \\
\text { demais esferas da saúde para } \\
\text { prevenir e identificar } \\
\text { precocemente o suicídio, } \\
\text { oferecer segurança tanto para } \\
\text { a pessoa com comportamentos } \\
\text { suicidas quanto para família e } \\
\text { estar pronto para atuar na } \\
\text { tentativa ou risco de suicídio. }\end{array}$ & $\begin{array}{l}\text { SANTOS, R. S.; } \\
\text { ALBUQUERQUE, M. } \\
\text { C. S.; BRÊDA, M. Z.; } \\
\text { BASTOS, M. L. A.; } \\
\text { SILVA, V. M. S.; } \\
\text { TAVARES, N. V. S. } \\
\text { (2017) }\end{array}$ \\
\hline $\begin{array}{l}\text { Ideação Suicida em } \\
\text { Jovens Adultos. }\end{array}$ & $\begin{array}{l}\text { Os profissionais que trabalham } \\
\text { frente a um grupo de risco, } \\
\text { devem entender melhor sobre } \\
\text { os fatores e problemáticas que } \\
\text { surgem no andar do } \\
\text { tratamento, para que assim, } \\
\text { eles possam ajudar e evitar que } \\
\text { o índice de suicídio tenha um } \\
\text { crescimento ainda maior. }\end{array}$ & $\begin{array}{lr}\text { SOARES, } & \text { R. } \\
\text { MENDES, L. A. } & \text { C.; } \\
\text { BRASILINO } & \text { E } \\
\text { ALENCAR, F. L. } & \\
& \\
(2018) & \end{array}$ \\
\hline $\begin{array}{l}\text { Fatores Associados à } \\
\text { Presença de Ideação }\end{array}$ & $\begin{array}{l}\text { Identificar os fatores que se } \\
\text { associam à presença de } \\
\text { ideação suicida nos estudantes }\end{array}$ & $\begin{array}{l}\text { DOS SANTOS, H. G. } \\
\text { B.; MARCON, S. R.; } \\
\text { ESPINOSA, M. M.; }\end{array}$ \\
\hline
\end{tabular}




\begin{tabular}{|c|c|c|}
\hline $\begin{array}{ll}\text { Suicida } & \text { entre } \\
\text { Universitários. } & \end{array}$ & $\begin{array}{l}\text { universitários pode constituir } \\
\text { uma importante ferramenta } \\
\text { para que ações de prevenção e } \\
\text { proteção sejam planejadas, } \\
\text { tanto por parte dos gestores da } \\
\text { universidade, como das } \\
\text { equipes de saúde que assistem } \\
\text { esses estudantes dentro e fora } \\
\text { do campus. }\end{array}$ & $\begin{array}{l}\text { BAPTISTA, M. N.; DE } \\
\text { PAULO, P. M. C. } \\
(2017)\end{array}$ \\
\hline $\begin{array}{lr}\text { Mortalidade } & \text { por } \\
\text { Suicídio } & \text { de } \\
\text { Adolescentes } & \text { no }\end{array}$ & $\begin{array}{l}\text { Os dados apresentados neste } \\
\text { estudo indicam que a } \\
\text { mortalidade de adolescentes }\end{array}$ & $\begin{array}{l}\text { CICOGNA, J. I. R.; } \\
\text { HILLESHEIM, } \\
\text { HALLAL, A. L. L. C. }\end{array}$ \\
\hline $\begin{array}{l}\text { Brasil: Tendência } \\
\text { Temporal de } \\
\text { Crescimento entre } \\
2000 \text { e 2015. }\end{array}$ & $\begin{array}{l}\text { por suicídio no Brasil } \\
\text { apresentou tendência de } \\
\text { crescimento nos últimos anos. } \\
\text { Destaca-se a importância de } \\
\text { que profissionais da saúde } \\
\text { estejam informados sobre } \\
\text { esses dados, para que possam } \\
\text { diagnosticar e intervir mais } \\
\text { cedo na prevenção do suicídio } \\
\text { em adolescentes. }\end{array}$ & \\
\hline $\begin{array}{l}\text { Uma Abordagem } \\
\text { Sobre o Suicídio de } \\
\text { Adolescentes } \\
\text { Jovens no Brasil. }\end{array}$ & $\begin{array}{l}\text { Apresenta argumentação sobre } \\
\text { a evolução das taxas de } \\
\text { mortalidade por suicídio } \\
\text { segundo as premissas } \\
\text { clássicas da saúde pública } \\
\text { sobre as causas sociais no } \\
\text { processo saúde-doença. }\end{array}$ & $\begin{array}{l}\text { RIBEIRO, J. M.; } \\
\text { MOREIRA, M. R. } \\
(2018)\end{array}$ \\
\hline $\begin{array}{l}\text { Do acolhimento ao } \\
\text { encaminhamento: }\end{array}$ & $\begin{array}{l}\mathrm{O} \text { adequado atendimento aos } \\
\text { pacientes que chegam aos }\end{array}$ & $\begin{array}{l}\text { DE FREITAS, A. P. A.; } \\
\text { BORGES, L. M. }\end{array}$ \\
\hline
\end{tabular}




\begin{tabular}{|c|c|c|}
\hline $\begin{array}{l}\text { atendimento às } \\
\text { tentativas de suicídio } \\
\text { nos contextos } \\
\text { hospitalares. }\end{array}$ & $\begin{array}{l}\text { serviços de saúde por tentativa } \\
\text { de suicídio é determinante para } \\
\text { a recuperação e prevenção de } \\
\text { que novas tentativas sejam } \\
\text { cometidas. }\end{array}$ & (2017) \\
\hline $\begin{array}{lr}\text { Suicídio na Voz de } \\
\text { Profissionais } \\
\text { Enfermagem de } \\
\text { Estratégias } \\
\text { Intervenção Diante do } \\
\text { Comportamento } \\
\text { Suicida. }\end{array}$ & $\begin{array}{l}\text { Os profissionais de } \\
\text { enfermagem devem estar } \\
\text { qualificados para realizar as } \\
\text { intervenções necessárias ao } \\
\text { paciente que procura o serviço } \\
\text { e para fornecer o } \\
\text { encaminhamento mais } \\
\text { adequado a cada caso. }\end{array}$ & $\begin{array}{l}\text { REISDORFER, N.; DE } \\
\text { ARAUJO, G. M.; } \\
\text { HILDEBRANDT, L. M.; } \\
\text { GEWEHR, R. T.; } \\
\text { NARDINO, J.; LEITE, } \\
\text { T. M. (2015) }\end{array}$ \\
\hline $\begin{array}{l}\text { Análise da mortalidade } \\
\text { por suicídio no Brasil } \\
1996 \text { a } 2015 .\end{array}$ & $\begin{array}{l}\text { Análise histórica da taxa de } \\
\text { suicídios no Brasil, de } 1996 \text { a } \\
\text { 2015, baseando-se em dados } \\
\text { secundários que estão } \\
\text { disponíveis no sítio do } \\
\text { Ministério da Saúde. O } \\
\text { conhecimento das taxas de } \\
\text { suicídio pode contribuir para o } \\
\text { delineamento de estratégias } \\
\text { preventivas a esses pacientes. }\end{array}$ & $\begin{array}{l}\text { BARROS, } \\
\text { (2018) }\end{array}$ \\
\hline $\begin{array}{l}\text { Epidemiologia do } \\
\text { suicídio no Brasil entre } \\
\text { os anos de } 2000 \text { e } \\
2010 \text {. }\end{array}$ & $\begin{array}{l}\text { Uma análise epidemiológica do } \\
\text { suicídio no Brasil, realizando } \\
\text { uma delimitação para identificar } \\
\text { se há obstáculos na conduta e } \\
\text { auxiliar na melhora das } \\
\text { estratégias para detecção, } \\
\text { conduta e prevenção do } \\
\text { suicídio. Um adequado registro }\end{array}$ & $\begin{array}{l}\text { FILHO, } \\
\text { ZERBINI, T. } \\
(2016)\end{array}$ \\
\hline
\end{tabular}




\begin{tabular}{|c|c|c|}
\hline & $\begin{array}{l}\text { garante maior foco e atenção } \\
\text { em meios efetivos de cuidados } \\
\text { e prevenção em pacientes } \\
\text { suicidas. }\end{array}$ & \\
\hline $\begin{array}{l}\text { Mindfulness and self } \\
\text { compassion: } \\
\text { exploring pathways to } \\
\text { adolescent emotional } \\
\text { well-being. }\end{array}$ & $\begin{array}{l}\text { Recognizing that mindfulness } \\
\text { brings awareness to one's } \\
\text { suffering and that self- } \\
\text { compassion addresses and } \\
\text { ameliorates that suffering, one } \\
\text { would expect that as one } \\
\text { becomes more mindful and } \\
\text { aware of her suffering, they } \\
\text { would initiate self- } \\
\text { compassionate and self- } \\
\text { comforting behavior. }\end{array}$ & $\begin{array}{l}\text { BLUTH, K.; BLANTON, } \\
\text { P. W. } \\
\text { (2013) }\end{array}$ \\
\hline $\begin{array}{l}\text { Suicídio entre crianças } \\
\text { e adolescentes, suas } \\
\text { principais causas e } \\
\text { métodos: Síntese de } \\
\text { evidências. }\end{array}$ & $\begin{array}{l}\text { Principais evidências } \\
\text { relacionadas ao suicídio entre } \\
\text { crianças e suas possíveis } \\
\text { causas, assim como a melhor } \\
\text { conduta a ser tomada pelo } \\
\text { pediatra ao lidar com esse tipo } \\
\text { de situação. }\end{array}$ & $\begin{array}{l}\text { BREDA, } \\
\text { GUERRA, P. } \\
\text { (2019) }\end{array}$ \\
\hline $\begin{array}{l}\text { Fatores associados à } \\
\text { ideação suicida na } \\
\text { adolescência: uma } \\
\text { revisão integrativa. }\end{array}$ & $\begin{array}{l}\text { Os adolescentes devem ser } \\
\text { alvos de estratégias de } \\
\text { prevenção, principalmente } \\
\text { quando já apresentaram } \\
\text { tentativas prévias de suicídio. } \\
\text { Campanhas educativas, com } \\
\text { foco no ambiente escolar, que } \\
\text { enfatizem a ideação suicida, } \\
\text { visando modificar o contexto }\end{array}$ & $\begin{array}{l}\text { NAVA, A. T. M.; } \\
\text { ALMEIDA, H. F. R.; } \\
\text { FONTENELE, R. M.; } \\
\text { RAMOS, A. S. M. B.; } \\
\text { CORTEZ, D. C. M.; } \\
\text { MONTEIRO, M. M. L. } \\
(2019)\end{array}$ \\
\hline
\end{tabular}




\begin{tabular}{|c|c|c|}
\hline & $\begin{array}{l}\text { em que estes jovens estão } \\
\text { inseridos e transformar os } \\
\text { educadores, a família e os } \\
\text { adolescentes em agentes } \\
\text { promotores de saúde. }\end{array}$ & \\
\hline $\begin{array}{l}\text { Revisão integrativa: } \\
\text { Conceitos e Métodos } \\
\text { Utilizados na } \\
\text { Enfermagem. }\end{array}$ & $\begin{array}{l}\text { Identificar e analisar os } \\
\text { conceitos atribuídos à Revisão } \\
\text { Integrativa e os métodos } \\
\text { recomendados para seu } \\
\text { desenvolvimento na área de } \\
\text { enfermagem. }\end{array}$ & $\begin{array}{lll}\text { SOARES, } & \text { C. } & \text { B.; } \\
\text { HOGA, L. A. } & \text { K.; } \\
\text { PEDUZZI, } & & \text { M.; } \\
\text { SANGALETI, } & \text { C.; } \\
\text { YONEKURA, } & \text { T.; } \\
\text { SILVA, D. R. A. D. } \\
(2014)\end{array}$ \\
\hline
\end{tabular}

Fonte: Elaborado pelos próprios autores desse estudo.

\section{DISCUSSÃO}

Os estudos incluídos na presente pesquisa perpassam a análise e interpretação dos resultados encontrados. Foram categorizados em três subcategorias: Suicídio entre os adolescentes no Brasil; Fatores Associados à Ideação Suicida na Adolescência e Acolhimento da Equipe de Enfermagem frente aos Adolescentes com Ideações Suicidas ou que Tentaram Suicídio, que estão apresentados a seguir.

\subsection{SUICÍDIO ENTRE ADOLESCENTES NO BRASIL}

O risco do suicídio pode ocorrer em qualquer faixa etária, mas atualmente vem crescendo de forma alarmante em adolescentes e jovens, que por sua vez, seriam pessoas mais instáveis e em busca de encontrar seu verdadeiro espaço, e viver livremente (SOARES, MENDES, ALENCAR, 2018).

Por estarem em uma fase de transição é comum que exista certa insegurança, conforme os anos vão passando, começam a identificar que as coisas não serão como antes, percebem que a jornada na idade da autonomia e independência, é mais difícil 
e cansativa, seja na vida profissional ou em quaisquer outras áreas que queiram buscar sua auto realização.

Devido a esse vasto quadro de mudanças na adolescência assim como a exposição a tantas outras situações complexas, é natural o desenvolvimento de um crescente sentimento de frustração e insatisfação pessoal, culminando em pensamentos que sejam desfavoráveis a vida. Os adolescentes com ideações suicidas são esses que buscam uma válvula de escape para tal situação, e idealizam planejamentos estratégicos que os retirem da realidade imediatamente (SOARES, MENDES, ALENCAR, 2018).

O suicídio pode ser definido como um ato deliberado executado pelo próprio indivíduo, cuja intenção seja a morte, de forma consciente e intencional, usando um meio que acredita ser letal. Também fazem parte do que habitualmente chamamos de comportamento suicida: os pensamentos, os planos e a tentativa de suicídio. (BIBLIOTECA VIRTUAL EM SAÚDE, 2018).

Visando melhorar e facilitar o entendimento da situação atual dos números de adolescentes com ideações suicidas no Brasil, foi realizado um levantamento das mortes por lesões autoprovocadas na faixa etária de 10 a 19 anos durante o período de 2000 a 2015 (CICOGNA, HILLESHEIM, HALLAL, 2019).

No Brasil ocorreram 11.947 mortes por lesões autoprovocadas intencionalmente em indivíduos de 10 a 19 anos no período supracitado (Gráfico 1). Essas mortes representam $8,25 \%$ do total de óbitos registrados por suicídio em todas as faixas etárias. A maior parte $(85,32 \%)$ dos suicídios na faixa etária estudada aconteceu com adolescentes de 15 a 19 anos. 
Gráfico 1 - Distribuição do número de óbitos por lesões autoprovocadas intencionalmente no Brasil na faixa etária de 10 a 19 anos no período de 2000 a 2015.

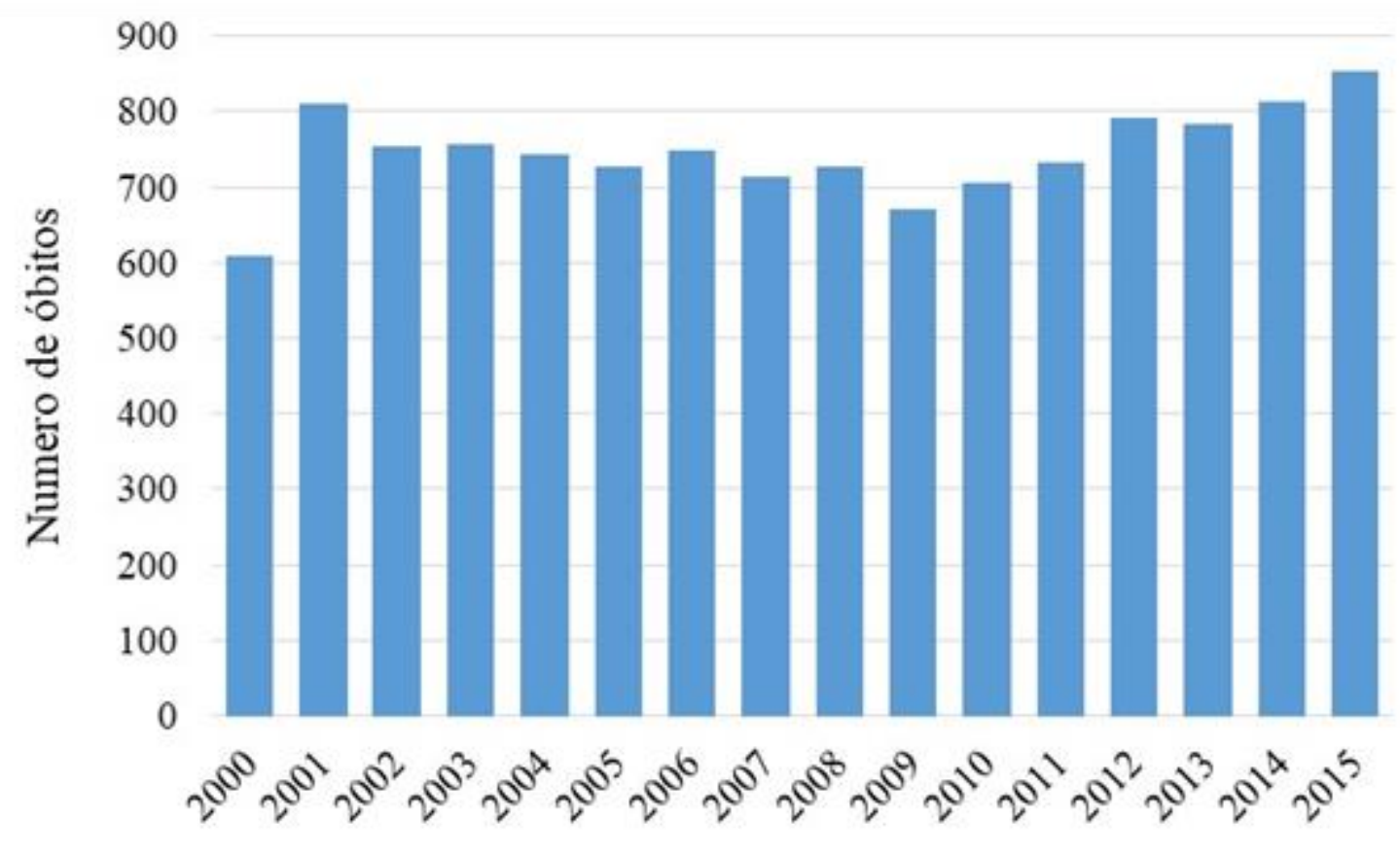

Ano

Fonte: CICOGNA, J. I. R.; HILLESHEIM, D.; HALLAL, A. L. L. C. Mortalidade por suicídio de adolescentes no Brasil: tendência temporal de crescimento entre 2000 e 2015. J Bras Psiquiatr, Florianópolis (SC), v. 68, n. 1, p. 1-7, 2019.

O método de suicídio mais utilizado foi o enforcamento, estrangulamento ou sufocação, responsável por $58,95 \%$ das mortes. O disparo de arma de fogo não especificada foi o segundo método mais frequentemente utilizado ( $9,75 \%$ das mortes). O terceiro método foi a auto-intoxicação por pesticidas (7,99\%). No ano 2000 , o coeficiente de mortalidade por lesões autoprovocadas intencionalmente na faixa etária de 10 a 19 anos em ambos os sexos no Brasil foi de 1,71 óbito a cada 100.000 habitantes, sendo que no ano 2015 esse coeficiente aumentou para 2,51. Esses resultados representam um crescimento de $47 \%$ na mortalidade por suicídio em adolescentes no Brasil (CICOGNA, HILLESHEIM, HALLAL, 2019). O Quadro 2 representa o coeficiente de mortalidade por lesões autoprovocadas intencionalmente em adolescentes de 10 a 19 anos por regiões brasileiras no período 
de 2000 a 2015. Na região Norte o índice de mortalidade por suicídio obteve um crescimento de $72,81 \%$, com uma variação de 2,28 a cada 100.000 habitantes no ano 2000 para 3,94 em 2015. Na região Nordeste, o índice aumentou de 1,14 a cada 100.000 habitantes no ano 2000 para 2,14 em 2015. Essa variação representou um aumento de $87,72 \%$. A região Centro-Oeste mostrou os maiores coeficientes de mortalidade por suicídio de adolescentes no Brasil. A região Sul foi a segunda região com mais mortes por suicídio no mesmo período. A região Centro-Oeste, apesar de não ter apresentado tendência de aumento, foi a região com maior taxa de óbitos por suicídio. Quando foi avaliado o Brasil como um todo, ocorreu oscilação nas taxas de óbitos por lesões autoprovocadas intencionalmente. Mesmo assim, o índice aumentou de 1,71 a cada 100.000 habitantes no ano 2000 para 2,51 em 2015 (CICOGNA, HILLESHEIM, HALLAL, 2019).

Quadro 2 - Coeficiente de mortalidade por lesões autoprovocadas intencionalmente de adolescentes de 10 a 19 anos por regiões brasileiras e o ano do óbito - Brasil, 2000 a 2015.

\begin{tabular}{|l|l|l|l|l|l|l|}
\hline & \multicolumn{2}{|l}{ Região } & & & \\
\hline Ano & Norte & Nordeste & Sudeste & Sul & Centro Oeste & Brasil \\
\hline 2000 & 2,28 & 1,14 & 1,14 & 3,01 & 4,27 & 1,71 \\
\hline 2001 & 2,89 & 1,47 & 2,06 & 3,82 & 3,52 & 2,29 \\
\hline 2002 & 2,55 & 1,58 & 1,69 & 3,50 & 3,87 & 2,13 \\
\hline 2003 & 2,56 & 1,64 & 1,75 & 3,29 & 3,98 & 2,16 \\
\hline 2004 & 2,42 & 1,70 & 1,57 & 3,43 & 4,21 & 2,13 \\
\hline 2005 & 2,49 & 1,75 & 1,45 & 3,21 & 4,48 & 2,10 \\
\hline 2006 & 2,34 & 1,85 & 1,74 & 3,16 & 3,69 & 2,16 \\
\hline 2007 & 3,04 & 1,98 & 1,52 & 2,59 & 3,15 & 2,07 \\
\hline 2008 & 3,10 & 2,00 & 1,40 & 2,98 & 3,44 & 2,11 \\
\hline 2009 & 3,39 & 1,71 & 1,17 & 2,73 & 3,70 & 1,95 \\
\hline 2010 & 3,17 & 1,96 & 1,47 & 2,58 & 3,02 & 2,05 \\
\hline 2011 & 3,14 & 1,87 & 1,74 & 2,66 & 2,92 & 2,13 \\
\hline 2012 & 3,02 & 1,95 & 1,77 & 3,06 & 4,19 & 2,31 \\
\hline
\end{tabular}




\begin{tabular}{|l|l|l|l|l|l|l|}
\hline 2013 & 3,83 & 2,01 & 1,53 & 3,17 & 3,67 & 2,29 \\
\hline 2014 & 3,73 & 2,08 & 1,63 & 3,62 & 3,44 & 2,38 \\
\hline 2015 & 3,94 & 2,14 & 1,83 & 3,09 & 4,45 & 2,51 \\
\hline
\end{tabular}

Fonte: CICOGNA, J. I. R.; HILLESHEIM, D.; HALLAL, A. L. L. C. Mortalidade por suicídio de adolescentes no Brasil: tendência temporal de crescimento entre $2000 \mathrm{e}$ 2015. J Bras Psiquiatr, Florianópolis (SC), v. 68, n. 1, p. 1-7, 2019.

Os casos de óbito entre adolescentes por suicídio no Brasil apresentaram tendência de crescimento nos últimos anos. Esses dados sugerem que os esforços nacionais para a prevenção do suicídio devem ser expandidos. Para que se aperfeiçoem as estratégias de prevenção ao suicídio, mais informações em relação aos fatores de risco específicos para adolescentes de cada região devem ser desenvolvidas. Destaca-se a importância de que profissionais da saúde, em especial os que trabalham nas regiões mais afetadas, estejam informados sobre esses dados, para que possam diagnosticar e intervir mais cedo nas ideações suicidas e na prevenção ao suicídio em adolescentes (CICOGNA, HILLESHEIM, HALLAL, 2019).

\subsection{FATORES ASSOCIADOS À IDEAÇÃO SUICIDA OU TENTATIVA DE SUICÍDIO NA ADOLESCÊNCIA}

Como já relatado anteriormente, entende-se por comportamento suicida, o suicídio consumado e a tentativa de suicídio. Pensamentos e planos suicidas são chamados ideação suicida. (CLAYTON, P. J., 2018)

Os fatores associados à ideação suicida na adolescência são multifacetados e incluem transtornos mentais, características pessoais e familiares, problemas comportamentais do próprio adolescente e dos amigos. Dentre os fatores que mais sobressaem destaca-se: depressão, desesperança, solidão, tristeza, preocupação, ansiedade, baixa autoestima, agressão por parte de pais e amigos, pouca comunicação com os pais, ser abusado fisicamente na escola (bulling), uso de substâncias psicoativas e ter pessoa em seu meio com tendências suicidas (MOREIRA e BASTOS, 2015). 
No Quadro 3 são apresentados diversos estudos realizados no Brasil com estudantes adolescentes, mostrando os fatores de desencadeamento da ideação suicida.

Quadro 3 - Fatores associados à ideação/risco suicida na adolescência no Brasil, 2002-2012.

\begin{tabular}{|c|c|c|}
\hline REFERÊNCIA/ANO & $\begin{array}{l}\text { FAIXA ETÁRIA DOS } \\
\text { ADOLESCENTES }\end{array}$ & $\begin{array}{ll}\text { FATORES } & \text { ALTAMENTE } \\
\text { SIGNIFICATIVOS } & \end{array}$ \\
\hline $\begin{array}{l}\text { FREITAS e BOTEGA } \\
(2002)\end{array}$ & $14 \mathrm{~A} 18$ anos & $\begin{array}{l}\text { Depressão, preocupação, pouco } \\
\text { apoio social. }\end{array}$ \\
\hline $\begin{array}{l}\text { WERLANG et al. } \\
(2005)\end{array}$ & 15 a 19 anos & $\begin{array}{l}\text { Depressão, conhecer pessoa } \\
\text { que tenha tentado suicídio. }\end{array}$ \\
\hline $\begin{array}{l}\text { BORGES e WERLANG } \\
\text { (2006a) }\end{array}$ & 15 a 19 anos & $\begin{array}{l}\text { Depressão, conhecer pessoa } \\
\text { que tenha tentado suicídio, } \\
\text { desesperança. }\end{array}$ \\
\hline $\begin{array}{l}\text { BORGES P WERLANG } \\
(2006 b)\end{array}$ & 15 a 19 anos & Depressão, sexo feminino. \\
\hline $\begin{array}{l}\text { JATOBÁ e BASTOS } \\
(2007)\end{array}$ & 14 a 16 anos & $\begin{array}{l}\text { Sintomas depressivos, } \\
\text { ansiedade. }\end{array}$ \\
\hline BORGES et al. (2008) & 13 a 17 anos & Depressão, sexo feminino. \\
\hline $\begin{array}{l}\text { BAGGIO, PALAZZO e } \\
\text { AERTS (2009) }\end{array}$ & 12 a 18 anos & $\begin{array}{l}\text { Agressão por parte dos pais e } \\
\text { colegas, solidão, depressão, } \\
\text { tristeza, poucos amigos } \\
\text { próximos. }\end{array}$ \\
\hline BOTEGA et al. (2009) & Idade $\geq 14$ anos & $\begin{array}{l}\text { Depressão, ansiedade, sexo } \\
\text { feminino, solteiro. }\end{array}$ \\
\hline ARAÚJO et al. (2010) & 14 a 18 anos & $\begin{array}{l}\text { Solidão, desesperança, } \\
\text { sofrimento psíquico. }\end{array}$ \\
\hline SOUZA et al. (2010a) & 15 a 18 anos & $\begin{array}{l}\text { Agressividade, sexo feminino, } \\
\text { consumo de álcool e drogas. }\end{array}$ \\
\hline SOUZA et al. (2010b) & 11 a 15 anos & $\begin{array}{l}\text { Depressão, sexo feminino, } \\
\text { consumo de álcool e drogas. }\end{array}$ \\
\hline
\end{tabular}


Fonte: MOREIRA, L. C. O.; BASTOS, P. R. H. O. Prevalência e fatores associados à ideação suicida na adolescência: revisão de literatura. Revista Quadrimestral da Associação Brasileira de Psicologia Escolar e Educacional, Campo Grande (MS), v. 19, n. 3, p. 445-453, 2015.

Os dados apresentados no Quadro 3 demonstram como é de suma importância a prevenção e o tratamento junto aos adolescentes com ideações suicidas, a assistência por parte da equipe de enfermagem é fundamental na redução dos números de novas tentativas e suicídios consumados.

\subsection{ACOLHIMENTO DA EQUIPE DE ENFERMAGEM FRENTE AOS ADOLESCENTES COM IDEAÇÕES SUICIDAS}

Com o acolhimento a equipe de enfermagem visa identificar as fragilidades emocionais desde o primeiro contato com esses pacientes, por meio da escuta ativa, podem reduzir significativamente novas tentativas de suicídios. Ao ouvir o usuário, os profissionais terão melhora na relação e desenvolvimento de uma parceria mais colaborativa (COSTA, GARCIA, TOLEDO, 2016).

Os profissionais não somente escutam ativamente essas informações, mas recebem e as interpretam, sendo verbais ou não verbais, e devolvem ao indivíduo com um novo significado, impactando diretamente na percepção sobre seu contexto vivido, logo, minimizando a chance de uma recaída, acarretando, simultaneamente na solidificação do relacionamento entre profissional, usuário e sistema de saúde (SANTOS et al., 2017).

O enfermeiro atuante no serviço de saúde deve estar qualificado e preparado para identificar os sinais de alerta com potencial suicida apresentado pelo adolescente, como pensamentos e atitudes que evidenciam desesperança, desespero e desamparo. Deve-se abordar o paciente de forma clara e cautelosa, mantendo a 
calma, empatia e abstendo-se das atitudes julgadoras, permitindo que o adolescente, de forma natural, inicie a criação de vínculo na medida em que a confiança evolui. $O$ acolhimento torna-se mais estruturado e com maiores chances de efetividade e, assim sendo, se desenvolverá um esforço conjunto para driblar as situações que o expõe as ideações suicidas e tentativas de suicídio.

Vale destacar que é absolutamente relevante respeitar sempre os limites dos adolescentes e chegar a um consenso sem ultrapassar a situação de vida, sentimentos e as necessidades que forem abordadas pelo adolescente, tornando real a chance de definirem juntos um acompanhamento rotineiro, ampliando o processo do cuidar para além do acolhimento. (BERTOLOTE, MELLO-SANTOS, BOTEGA, 2010).

\section{CONSIDERAÇÕES FINAIS}

Diante do exposto, concluiu-se que a ideação suicida em adolescentes apresenta tendências de crescimento nos últimos anos. Os dados apresentados neste estudo indicam que o coeficiente de mortalidade por lesões autoprovocadas intencionalmente em adolescentes aumentou nos últimos 20 anos em todas as regiões do Brasil, sendo que as regiões Centro-Oeste e Sul foram as que apresentaram os maiores coeficientes de mortalidade por suicídio.

A importância de se estudar a ideação suicida reside no fato de que ela é um dos gatilhos para o risco de suicídio, podendo ser o primeiro passo para sua consumação. É importante destacar que os profissionais da enfermagem precisam estar preparados para prestar assistência adequada aos adolescentes com ideações suicidas ou após tentativa de suicídio, e aos familiares, por meio da escuta ativa, autorreflexão e comunicação terapêutica, providenciando a assistência necessária imediata de forma humanizada.

A presença da ideação suicida sempre deve ser considerada como fator importante, e deve ser identificada no primeiro contato do profissional com o paciente, caso o problema não seja tratado de maneira adequada, o adolescente poderá cometer 
suicídio pelo fato de não ter tido apoio e o acolhimento correto por parte da equipe de enfermagem. Dessa forma, faz-se necessário que programas e estratégias de prevenção dos comportamentos suicidas sejam incluídos na pauta das políticas de educação e saúde pública. É primordial que o enfermeiro seja devidamente treinado e capacitado periodicamente sobre o tema em questão, uma vez que a perda prematura de adolescentes por suicídio pode e deve ser evitada.

\section{REFERENCIAS}

ARAÚJO, L. C.; VIEIRA, K. F. L.; COUTINHO, M. P. L. Ideação suicida na adolescência: um enfoque psicossociológico no contexto do ensino médio. Psicologia - Universidade São Francisco, João Pessoa (PB), v. 15, n. 1, p. 47-57, 2010.

BAGGIO, L.; PALAZZO, L. S.; AERTS, D. R. G. C. Planejamento suicida entre adolescentes escolares: prevalência e fatores associados. Cadernos de Saúde Pública, Porto Alegre (RS), v. 25, n. 1, p. 142-150, 2009.

BARROS, M. V. M. Análise da mortalidade por suicídio no Brasil 1996 a 2015. Trabalho de Conclusão de Curso (Especialização em Residência Multiprofissional em Saúde Coletiva). Instituto Aggeu Magalhães - Fundação Oswaldo Cruz, Recife (PE), 2018. Disponível em: <https://www.arca.fiocruz.br/handle/icict/29894>. Acesso em: 10 out. 2019.

BERTOLOTE, J. M.; MELLO-SANTOS, C.; BOTEGA, N. J. Detecção do risco de suicídio nos serviços de emergência psiquiátrica. Revista Brasileira de Psiquiatria, São Paulo (SP), v. 32, p. S87-S95, 2010.

BIBLIOTECA VIRTUAL EM SAÚDE. Setembro Amarelo e Dia Mundial da Prevenção ao Suicídio. 2018.

Disponível em: <bvsms.saude.gov.br/componente/contente/article?id=2787>. Acesso em: 07/08/2019.

BLUTH, K.; BLANTON, P. W. Mindfulness and self-compassion: exploring pathways to adolescent emotional well-being. Journal of child and family studies [Internet], EUA, 
v. $23, \quad$ n. $7, \quad$ p. 1298-309, 2013. Disponível em: <http://link.springer.com/article/10.1007/s10826-013-9830-2>. Acesso em: 12/10/2019.

BORGES, V. R.; WERLANG, B. S. G. Estudo de ideação suicida em adolescentes de 15 a 19 anos. Estudos de Psicologia, Porto Alegre (RS), v. 11, n. 3, p. 345-351, 2006 a.

BORGES, V. R.; WERLANG, B. S. G. Estudo de ideação suicida em adolescentes de 13 a 19 anos. Psicologia, Saúde e Doenças, Porto Alegre (RS), v. 7, n. 2, p. 195-209, 2006b.

BORGES, V. R.; WERLANG, B. S. G.; COPATTI, M. Ideação suicida em adolescentes de 13 a 17 anos. Barbarói, Erechim (RS), v. 28, p. 109-123, 2008.

BOTEGA, N. J.; MARÍN-LEÓN, L.; OLIVEIRA, H. B.; BARROS, M. B. A.; SILVA, V. F.; DALGALARRONDO, P. Prevalências de ideação, plano e tentativa de suicídio: um inquérito de base populacional em Campinas, São Paulo, Brasil. Cadernos de Saúde Pública, Campinas (SP), v. 25, n. 12, p. 2632-2638, 2009.

BREDA, L. C.; GUERRA, P. Suicídio entre crianças e adolescentes, suas principais causas e métodos: Síntese de evidências. Revista UNILUS Ensino e Pesquisa, Santos (SP), v. 16, n. 42, p.39-49,2019.

CLAYTON, J. P., Manual MSD - Versão Saúde para a Família, Comportamento Suicida. University of Minnesota School of Medicine, 2018. Disponível em:<https://www.msdmanuals.com/pt-br/casa/dist\%C3\%BArbios-de-sa\%C3\%BAdemental/comportamento-suicida-e-automutila\%C3\%A7\%C3\%A3o/comportamentosuicida>. Acesso em: 20/09/2019.

CICOGNA, J. I. R.; HILLESHEIM, D.; HALLAL, A. L. L. C. Mortalidade por suicídio de adolescentes no Brasil: tendência temporal de crescimento entre 2000 e 2015. J Bras Psiquiatr, Florianópolis (SC), v. 68, n. 1, p. 1-7, 2019. 
COSTA, Paula Cristina Pereira da; GARCIA, Ana Paula Rigon Francischetti; TOLEDO, Vanessa Pellegrino. Acolhimento e cuidado de enfermagem: Um estudo fenomenológico. Texto contexto - enferm, Florianópolis (SC), v. 25, n. 1, e4550015, p. 1-7, 2016. Disponível em: <http://www.scielo.br/pdf/tce/v25n1/pt_0104-0707-tce-2501-4550015.pdf>. Acesso em: 25/09/2019.

DE FREITAS, A. P. A.; BORGES, L. M. Do acolhimento ao encaminhamento: O atendimento às tentativas de suicídio nos contextos hospitalares. Estudos de psicologia, Santa Catarina (SC), v. 22, n. 1, p. 1678-4669, 2017.

DOS SANTOS, H. G. B.; MARCON, S. R.; ESPINOSA, M. M.; BAPTISTA, M. N.; DE PAULO, P. M. C. Fatores associados à presença de ideação suicida entre universitários. Rev. Latino-Am. Enfermagem, Cuiabá (MT), v. 25, e2878, 2017.

FILHO, M. C.; ZERBINI, T. Epidemiologia do suicídio no Brasil entre os anos de 2000 e 2010. Saúde, Ética \& Justiça, São Paulo (SP), v. 21, n. 2, p. 45-51, 2016.

FREITAS, G. V. S.; BOTEGA, N. J. Gravidez na adolescência: prevalência de depressão, ansiedade e ideação suicida. Revista da Associação Médica Brasileira, Campinas (SP), v. 48, n. 3, p. 245-249, 2002.

JATOBÁ, J. A. V. N. e BASTOS, O. Depressão e ansiedade em adolescentes de escolas públicas e privadas. Jornal Brasileiro de Psiquiatria, Recife (PE), v. 56, n. 3, p. 171-179, 2007.

MOREIRA, L. C. O.; BASTOS, P. R. H. O. Prevalência e fatores associados à ideação suicida na adolescência: revisão de literatura. Revista Quadrimestral da Associação Brasileira de Psicologia Escolar e Educacional, Campo Grande (MS), v. 19, n. 3, p. 445-453, 2015.

NAVA, A. T. M.; ALMEIDA, H. F. R.; FONTENELE, R. M.; RAMOS, A. S. M. B.; CORTEZ, D. C. M.; MONTEIRO, M. M. L. Fatores associados à ideação suicida na adolescência: uma revisão integrativa. Rev Enferm UFPI, São Luís (MA), v. 8, n. 2, p. 66-73, 2019. 
ORES, L. C.; QUEVEDO, L. A.; JANSEN, K.; CARVALHO, A. B.; CARDOSO, T. A.; SOUZA, L. D. M.; PINHEIRO, R. T.; SILVA, R. A. Risco de suicídio e comportamentos de risco à saúde em jovens de 18 a 24 anos: um estudo descritivo. Cadernos de Saúde Pública, Pelotas (RS), v. 28, n. 2, p. 305-312, 2012.

ORGANIZAÇÃO MUNDIAL DE SAÚDE (OMS). Folha Informativa - Suicídio. 2018. Disponível

em:

$<$ https://www.paho.org/bra/index.php?option=com_content\&view=article\&id=5671:fol ha-informativa-suicidio\&ltemid=839>. Acesso em: 03/08/2019.

REISDORFER, N.; DE ARAUJO, G. M.; HILDEBRANDT, L. M.; GEWEHR, R. T.; NARDINO, J.; LEITE, T. M. Suicídio na Voz de Profissionais de Enfermagem e Estratégias de Intervenção Diante do Comportamento Suicida. Revista de enfermagem da UFSM, Palmeira das Missões (RS), v. 5, n. 2, p. 295-304, 2015.

RIBEIRO, J. M.; MOREIRA, M. R. Uma abordagem sobre o suicídio de adolescentes e jovens no Brasil. Ciência saúde coletiva, Rio de Janeiro (RJ), v. 23, n. 9, p. 2821 2834, 2018.

SANTOS, R. S.; ALBUQUERQUE, M. C. S.; BRÊDA, M. Z.; BASTOS, M. L. A.; SILVA, V. M. S.; TAVARES, N. V. S. A atuação do enfermeiro com a pessoa em situação de suicídio: análise reflexiva. Revista de Enfermagem UFPE, Recife (PE), v. 11, n. 2, p. 742-748, 2017.

SOARES, C. B.; HOGA, L. A. K.; PEDUZZI, M.; SANGALETI, C.; YONEKURA, T.; SILVA, D. R. A. D. Revisão integrativa: conceitos e métodos utilizados na enfermagem. Rev. esc. enferm. USP [Internet], São Paulo (SP), v. 48, n. 2, p. 335345 , 2014. Disponível em:

$<$ http://www.scielo.br/scielo.php?script=sci_arttext\&pid=S008062342014000200335\&lng=en\&nrm=iso >. Acesso e: 13/10/2019.

SOARES, R. F.; MENDES, L. A. C.; BRASILINO E ALENCAR, F. L. Ideação suicida em jovens adultos. Conbracis. 2018. Disponível em 
<http://editorarealize.com.br/revistas/conbracis/trabalhos/TRABALHO_EV108_MD4_ SA13_ID1526_21052018205819.pdf>. Acesso em: 01/09/2019.

SOUZA, L. D. M.; ORES, L.; OLIVEIRA, G. T.; CRUZEIRO, A. L. S.; SILVA, R. A.; PINHEIRO, R. T.; HORTA, B.L. Ideação suicida na adolescência: prevalência e fatores associados. Jornal Brasileiro de Psiquiatria, Pelotas (RS), v. 59, n. 4, p. 286-292, 2010a.

SOUZA, L. D. M.; SILVA, R. A.; JANSEN, K.; KUHN, R. P.; HORTA, B. L.; PINHEIRO, R. T. Suicidal ideation in adolescents aged 11 to 15 years: prevalence and associated factors. Revista Brasileira de Psiquiatria, Pelotas (RS), v. 32, n. 1, p. 37-41, $2010 \mathrm{~b}$.

VABO, A. S. R.; CONRAD, D.; BAPTISTA, C.; AGUIAR, B. G. C.; FREITAS, V. L.; PEREIRA, G. L. Comportamento suicida: um olhar para além do modelo biomédico. Revista ACRED, Rio de Janeiro (RJ), v. 6, n. 12, p. 66-83, 2016.

WERLANG, B. S. G.; BORGES, V. R.; FENSTERSEIFER, L. Fatores de risco ou proteção para a presença de ideação suicida na adolescência. Revista Interamericana de Psicologia, Porto Alegre (RS), v. 39, n. 2, p. 259-266, 2005.

Enviado: Novembro, 2019.

Aprovado: Novembro, 2019. 